\title{
Fuzzy Region Merging Using Fuzzy Similarity Measurement on Image Segmentation
}

\author{
Wawan Gunawan ${ }^{1}$, Agus Zainal Arifin ${ }^{2}$, Rarasmaya Indraswari ${ }^{3}$, Dini Adni Navastara ${ }^{4}$ \\ ${ }^{1}$ Department of Science, Informatic Engineering, Institut Teknologi Sumatera, Bandar Lampung, Indonesia \\ ${ }^{2,3,4}$ Departement of Informatics, Institut Teknologi Sepuluh Nopember, Surabaya, Indonesia
}

\begin{tabular}{l}
\hline Article Info \\
\hline Article history: \\
Received Apr 8, 2017 \\
Revised Sep 8, 2017 \\
Accepted Sep 25, 2017 \\
\hline
\end{tabular}

\section{Keyword:}

Ambiguous region Image segmentation Fuzzy region merging

\begin{abstract}
Some image's regions have unbalance information, such as blurred contour, shade, and uneven brightness. Those regions are called as ambiguous regions. Ambiguous region cause problem during region merging process in interactive image segmentation because that region has double information, both as object and background. We proposed a new region merging strategy using fuzzy similarity measurement for image segmentation. The proposed method has four steps; the first step is initial segmentation using mean-shift algorithm. The second step is giving markers manually to indicate the object and background region. The third step is determining the fuzzy region or ambiguous region in the images. The last step is fuzzy region merging using fuzzy similarity measurement. The experimental results demonstrated that the proposed method is able to segment natural images and dental panoramic images successfully with the average value of misclassification error (ME) $1.96 \%$ and $5.47 \%$, respectively.
\end{abstract}

Copyright () 2017 Institute of Advanced Engineering and Science. All rights reserved.

\section{Corresponding Author:}

Wawan Gunawan,

Department of Science, Informatics Engineering,

Institut Teknologi Sumatera,

Bandar Lampung, Indonesia.

Email: wawan.gunawan@if.itera.ac.id

\section{INTRODUCTION}

Segmentation is a basic process in image processing. The purpose of segmentation is to divide the image into regions that have homogenous features or have the same characteristics, e.g., contours, colors, and contrast [1],[2]. In general, image segmentation methods can be divided among three categories, namely automatic, semi-automatic, and manual [3]. Automatic image segmentation methods can be categorized into several groups, namely the histogram-based, edge-based, region-based [4],[5], and hybrid technique [6]. Although automatic segmentation method is fast, optimization process needs to be done to get the optimal parameters that greatly affect the accuracy of automatic segmentation results [7].

Automatic segmentation methods have drawbacks when the object and the background region of the image did not have a clear dividing line, causing a difference in perception between the results of the segmentation method and the user's wishes [8]. Semi-automatic segmentation method has been developed to overcome that problem by providing additional information from the user to assist the system in the segmentation process. Under these conditions, our study used a semi-automatic segmentation approach or often referred to as the interactive image segmentation.

In interactive image segmentation, user can interact by providing input (user marking) that helps the system in the determination of the object and the background area in the image. Several studies related to semi-automatic segmentation have been proposed by [3],[9]-[12]. Based on those study, interactive image segmentation consist of four main stages. The first stage is dividing the image into several small regions (region splitting) to get the initial segmentation. The second stage is user marking manually some regions as 
object or background. The third stage is the extracting each region based on its features, such as color, shape, membership function, texture, or size of the region. The last stage is merging all regions, to get two clusters which are background and object.

Some regions have unbalance information values, such as blurred contour, shade, and uneven brightness. In this study, we refer those regions as ambiguous regions. The ambiguous region is very influential in the process of region splitting because they are very similar hence it is difficult to separate them. The ambiguous region will be considered a single region even though the region has two values of information, which are objects and background information. This can lead to error during the region merging process for causing over segmentation. Figure 1(A) is an example of the ambiguous region, we can see that the color in the region is very similar (fuzzy region) so it would be difficult to separate the region [13]-[14]. In Figure 1(B), although those two regions that have similar color, there is a clear line between those regions hence it will be easy to separate them.


Figure 1. Different transition color in the region. (A) The ambiguous region, (B) Non -ambiguous region

The ambiguous region will affect the region merging process because the ambiguous region caused over segmentation in the region splitting process. In binary region merging (BRM) [10],[15] each region has only one probability (crisp fuzzy) to be in the object or background cluster. For images that have an ambiguous region, binary region merging cannot be done because the region has two information values.

In this study, we propose a new strategy for region merging, namely fuzzy region merging, using fuzzy similarity measurement in interactive image segmentation. Our contribution to this research is the fuzzy region merging (FRM) process where each region will be merged using fuzzy similarity measurements, so ambiguous regions within the image can be separated.

\section{RESEARCH METHOD}

Input images that are used for this study are natural images and dental panoramic images. The natural images are obtained from real-world objects with different backgrounds and objects. Dental panoramic images are obtained from Airlangga University Hospital [16]. Overall, we used grayscale images. In this study, we focused just on the region merging strategy to overcome the ambiguous regions on the image. We find the optimal similarity between regions using fuzzy similarity measurement. The steps of our proposed method can be seen in Figure 2.



Figure 2. Stages of the proposed method

\subsection{Initial Segmentation}

Initial segmentation aims to divide the image into several small regions that share similar characteristics. In this study, to get initial segmentation we use mean-shift segmentation software created by 
Edison System. The image is divided into several regions based upon the probability density gradient functions. The result of the initial segmentation using the mean-shift algorithm is better than other methods of low-level segmentation, because it is considering the spatial information and shape of the object image [10].

\subsection{Markers}

Interactive image segmentation provides user interaction with the segmentation system in the form of markers. Manual marking is one of the most major stages in the interactive segmentation because it will affect the segmentation result. Interactive image segmentation is very sensitive to the quality of marking and the number of marker [17]. Figure 3 illustrates the region marking process for natural and dental panoramic images, the green line indicates the object region and the blue line indicates the background region. The features of the regions that has been marked as object or background is carried out to determine its characteristics.
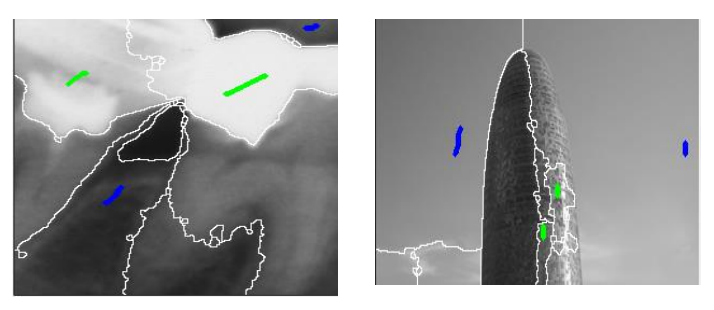

Figure 3. Results of initial segmentation and user marking

\subsection{Initialization of Fuzzy Region}

Each member of the fuzzy set has a degree of membership value that determines the potential members can enter a fuzzy. This stage is used to find the fuzzy region in the image, where the parameters of each region that has been marked as the object $M_{O}$ and background $M_{B}$ will be calculated. Parameter obtained by finding the highest gray level at each marker of region background $M_{b}^{(f)}$ and from the smallest gray level at each marker of object region $M_{o}^{(f)}$. $M_{B}$ value will always smaller than the value of $M_{O}$. The value of $M_{B}$ and $M_{O}$ is calculated using Eq. 1-3. Figure 4 shows the illustration of the determination of $M_{b}^{(f)}$ and $M_{o}^{(f)}$ parameters to describe the value of $M_{B}$ and $M_{O}$. Fuzzy region is an ambiguous region of the image which intensity is always between $M_{B}$ and $M_{O}$. Initial seed of background region $C_{B}$ is the area between $M_{B}$ and the minimal gray level in the histogram. Initial seed of object region $C_{O}$ is the area between $M_{O}$ and the maximal gray level on the histogram.

$$
\begin{aligned}
& M_{B}=\max \left(g ; M_{b}^{(f)}\right) \\
& M_{O}=\min \left(g ; M_{o}^{(f)}\right) \\
& f(x)= \begin{cases}M_{B}=M_{O} ; M_{O}=M_{B}, V_{B}>V_{O} \\
M_{B}=M_{B} ; M_{O}=M_{O}, \text { otherwise }\end{cases}
\end{aligned}
$$

After the fuzzy region was found, the next step is calculate the membership function in the gray level histogram. S-function calculates the background membership function $\mu_{B}$ and Z-function calculates the object membership function $\mu_{o}$. Each membership function is controlled by a point $M_{C}=127$ and is calculated using Eq. 4 and Eq. 5. Figure 5 illustrates $S$-function that forms the letter $S$ with a green line on the histogram. 


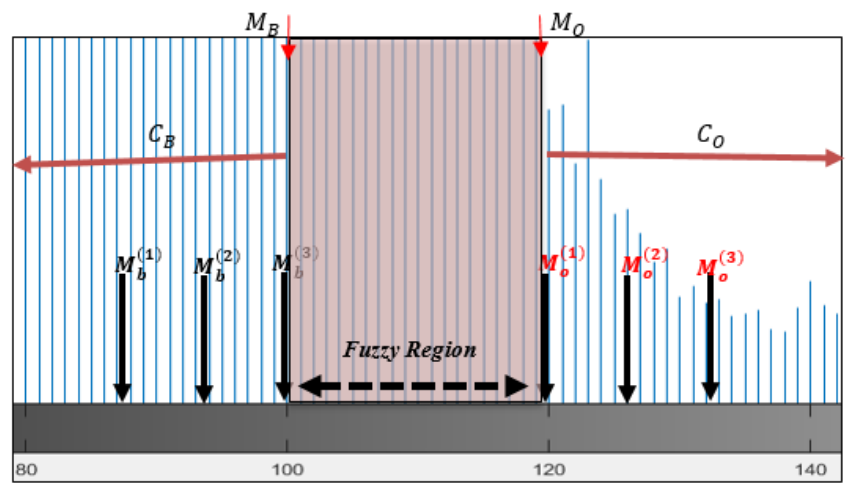

Figure 4. Initializes the fuzzy region in the histogram

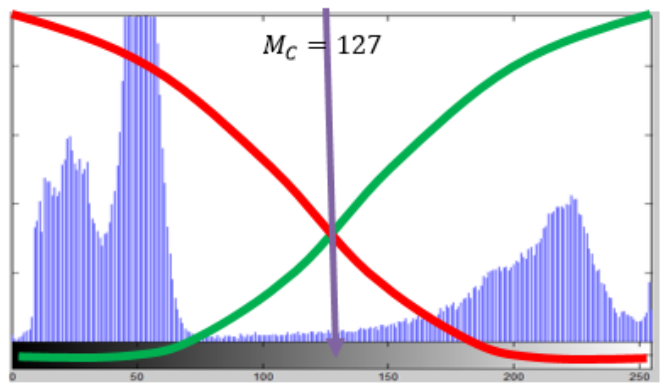

Figure 5. Determination of S-function and Z-function in the gray level histogram

The smaller the value of gray level in the histogram, the greater the membership function of background in the histogram. Z-function forms the letter $\mathrm{Z}$ with red line on the histogram. The larger the value of gray level in the histogram, the greater the gray level membership function of object in the histogram. We use S-function and Z-function because these functions consider the membership function of the object and the background object also against a contradictory background.

$$
\begin{aligned}
& \mu_{B}(g)=S\left(g ; M_{B}, M_{C}, M_{O}\right)= \begin{cases}0, & g<M_{B} \\
2\left\{\frac{g-M_{B}}{M_{O}-M_{B}}\right\}^{2}, & M_{B} \leq g \leq M_{C} \\
1-2\left\{\frac{g-M_{O}}{M_{O}-M_{B}}\right\}^{2}, & M_{C}<g \leq M_{O} \\
1, & g>M_{O}\end{cases} \\
& \mu_{O}(g)=1-S\left(g ; M_{B}, M_{C}, M_{O}\right)
\end{aligned}
$$

\subsection{Fuzzy Region Merging}

The last stage is fuzzy region merging for each fuzzy region $f_{i \ldots . . .} \in F$. We use fuzzy similarity measurement on initial seed of background region $C_{B}$ and initial seed of object region $C_{O}$. Fuzzy similarity calculated based on the similarity between the gray level and the intensity, membership functions, and the difference of membership function with the ordinal set. Fuzzy similarity measurement $\delta$ calculates the initial subset of global information $C_{B}$ and $C_{O}$ to local information on each fuzzy region $f_{i}$ in the image as illustrated in Figure 6. Similarity value $\delta$ for set $\left(C_{X} \cup\left\{f_{i g}\right\}\right)$, initial seed of an area $C_{X}$, membership of all $g$ gray level in the fuzzy region $\mathrm{f}_{\mathrm{i}}$, and gray level intensity $\mathrm{h}(\mathrm{g})$, can be calculated using Eq. 6 .

$$
\left(C_{\mathrm{x}} \cup\left\{\mathrm{f}_{\mathrm{ij}}\right\}\right)=\frac{\sum_{\mathrm{g}=1}^{\mathrm{n}}\left(\mathrm{g}-\mathrm{fm}\left(\mathrm{C}_{\mathrm{x}} \cup\left\{\mathrm{f}_{\mathrm{ig}}\right\}\right)^{2}\right.}{\sum_{\mathrm{z}=1}^{\mathrm{n}} \mathrm{h}(\mathrm{g})}
$$




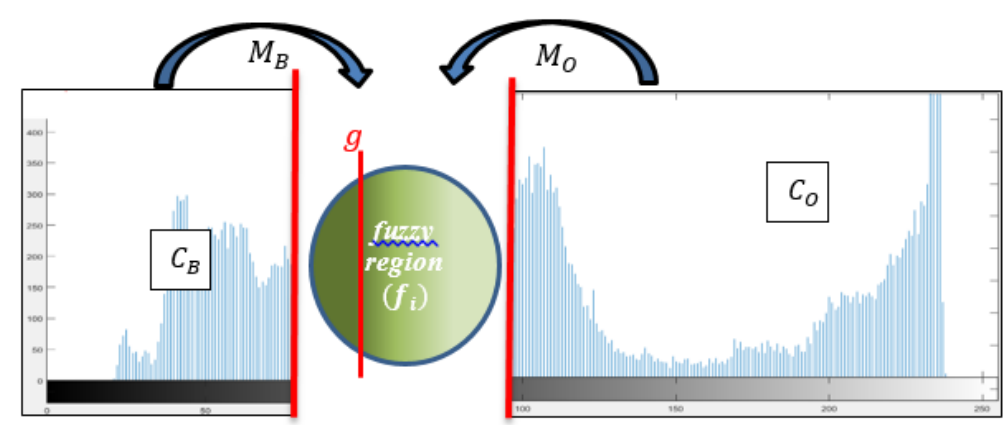

Figure 6. Fuzzy region merging using fuzzy similarity measurement

Fuzzy mean value $f m(A)$ of the merged area $A$, that is considering the gray level intensity $h(g)$, membership functions $\mu_{A}(g)$, and the difference of membership function with the ordinal set $\mid\left(\mu_{\mathrm{A}}(\mathrm{g})-\right.$ $\mu_{A}^{\prime}(\mathrm{g}) \mid$, can be calculated using Eq. 7. Based on the results of fuzzy similarity measurement, each fuzzy region $f_{i g}$ can be merged to background or object cluster based on the greatest similarity of the fuzzy region. Determining the similarity value $g$ in fuzzy region $\delta_{i g}$ can be calculated using Eq. 8 by finding the largest index.

$$
\begin{aligned}
& P(A)=\sum_{z=1}^{n} h(g) \times \mu_{A}(g) \times\left|\left(\mu_{A}(g)-\mu_{A}^{\prime}(g)\right)\right| . \\
& \delta_{i g}=\operatorname{argmax}\left(\delta\left(C_{B} \cup\left\{f_{i j}\right\}\right) * \delta\left(C_{O} \cup\left\{f_{i j}\right\}\right)\right)
\end{aligned}
$$

\section{EXPERIMENTAL RESULT}

The proposed method is implemented on the 15 dental panoramic images (that have been used in [12] and [16]) and 10 natural images. Figure 7 show several of the test images after initial segmentation and user marking process. Figure 8 shows the ground truth images that are created manually. Figure 9 shows the segmentation results of the proposed method. We also compared our proposed method with binary region merging approach proposed by Ning et.al. [10], named maximal similarity based region merging (MSRM).

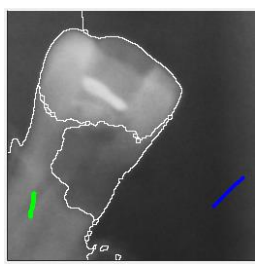

(a)

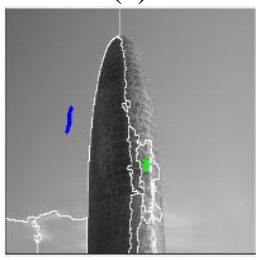

(e)

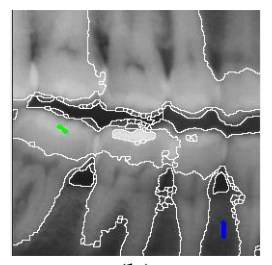

(b)

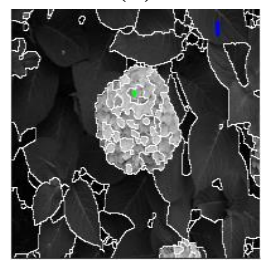

(f)

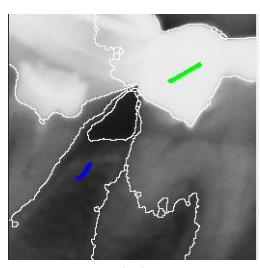

(c)

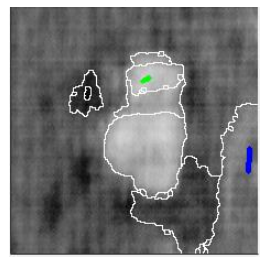

(g)

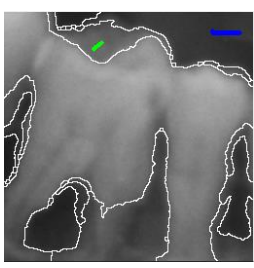

(d)

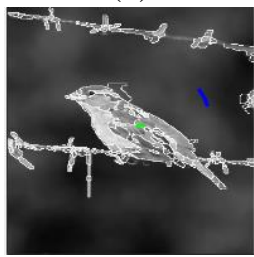

(h)

Figure 7. Sample of input images 


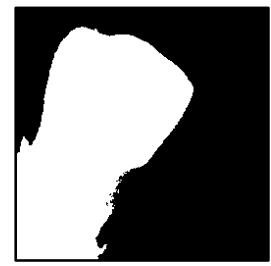

(a)

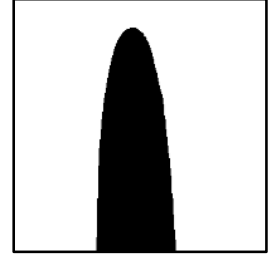

(e)

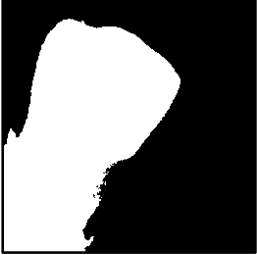

(a)

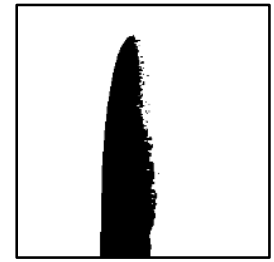

(e)

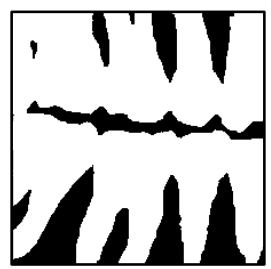

(b)

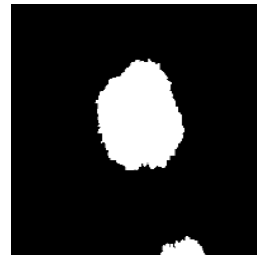

(f)

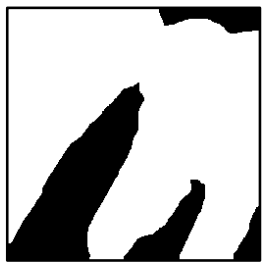

(c)

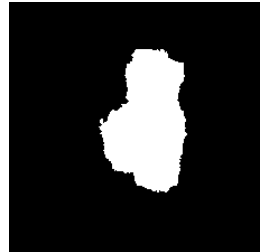

(g)

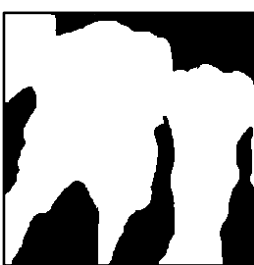

(d)

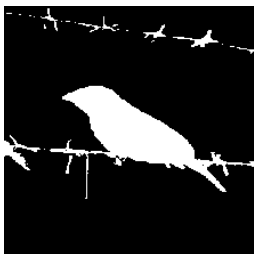

(h)

Figure 8. Ground truth images

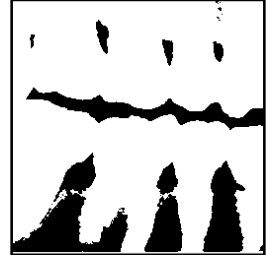

(b)

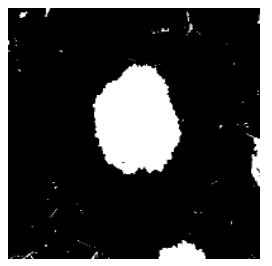

(f)

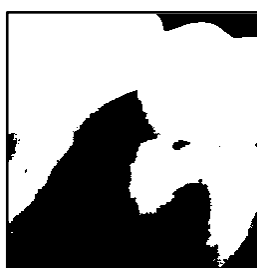

(c)

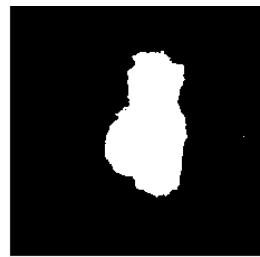

(g)

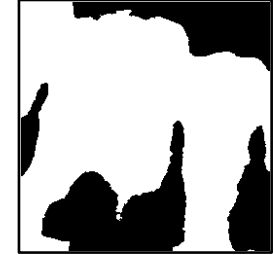

(d)

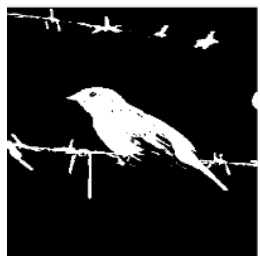

(h)

Figure 9. Segmentation results of the proposed method

Segmentation results of MSRM are shown in Figure 10. Each segmented image will be compared with ground truth images to determine the performance of segmentation results. In this study, the evaluation was conducted by using misclassification error (ME) that is calculated based on the Eq. 9.

$$
M E=1-\frac{\left|B_{O} \cap B_{T}\right|+\left|F_{O} \cap F_{T}\right|}{\left|B_{O}\right|+\left|F_{O}\right|}
$$

where $B_{O}$ and $F_{O}$ are the background and the object of the ground truth image, while $B_{T}$ and $F_{T}$ are the background and the object of the segmentation result. The smaller value of ME shows the segmentation results method is getting better and closer to ground truth images.

The implementation results based on the value of ME for several of the test images is shown in Table 1. The proposed method provides better performance than MSRM with an average ME value $4.55 \%$ for natural images and $5.46 \%$ for dental panoramic images. It was concluded that the proposed method is more resistant to the interference of ambiguous region. 


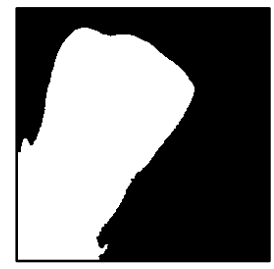

(a)

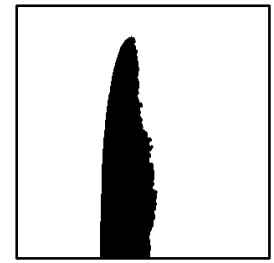

(e)

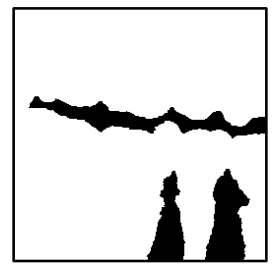

(b)

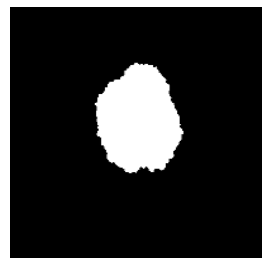

(f)

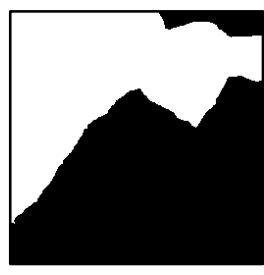

(c)

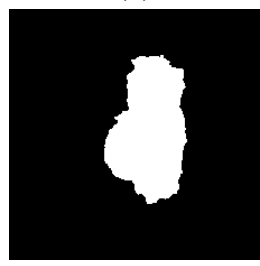

$(\mathrm{g})$

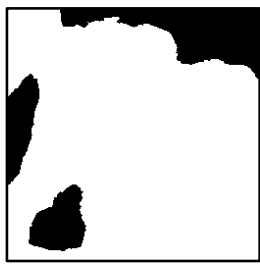

(d)

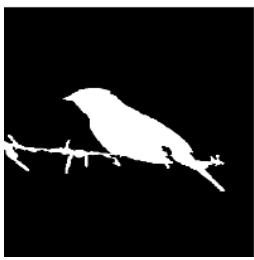

(h)

Figure 10. Segmentation results of the MSRM method

Table 1. Comparison result of the proposed method and MSRM on several test images

\begin{tabular}{cccc}
\hline \multirow{2}{*}{ No } & Images & \multicolumn{2}{c}{ Misclassification Error (\%) } \\
& & MSRM & Proposed method \\
\hline a & Dental 1 & 1.97 & 2.38 \\
b & Dental 2 & 15.64 & 9.42 \\
c & Dental 3 & 33.47 & 14.38 \\
d & Dental 4 & 17.37 & 7.70 \\
e & Natural 1 & 7.60 & 7.52 \\
f & Natural 2 & 1.11 & 0.77 \\
g & Natural 3 & 0.91 & 0.98 \\
h & Natural 4 & 2.48 & 3.16 \\
\hline
\end{tabular}

\section{DISCUSSION}

Based on the experimental results, the discussion of this study is divided into 3 sections. Section 4.1 will discuss about the initial segmentation process using the mean-shift algorithm. Section 4.2 analyzes user marking process. And Section 4.3 will analyze the fuzzy region merging.

\subsection{Experiment Analysis of Region Splitting}

Region Splitting using mean-shift software from Edison system has been successfully implemented to get the initial segmentation. The image can be divided into several homogeneous regions. Some studies also used the mean-shift software to get the initial segmentation as proposed by [3],[9]-[12]. There are two parameters that must be entered for this application is spatial bandwidth (sb) and the color bandwidth (cb). In this study, we test the spatial bandwidth values between 7-20 for natural images and 20-50 for dental panoramic images. For color bandwidth, we use value of 3.5-6.5 for natural images and 4.5-5.5 for dental panoramic images.

On image that has an ambiguous region, it is very difficult to get the right parameters to obtain the initial segmentation. Figure 11 shows the example of initial segmentation with different parameters. The images in the first row on Figure 11 shows that there will be different initial segmentation result using different parameters. However, over segmentation is happened on those results because there is ambiguous elapsed areas within the region. This is unlike the initial segmentation results for the images in the second row on Figure 11. It can be concluded that the ambiguous region will be very influential at the time of the initial segmentation and will certainly affect the results of segmentation. 


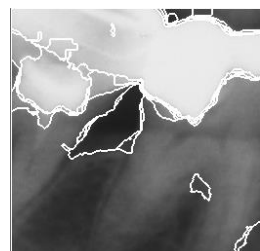

$(\mathrm{sb}=7$ and $\mathrm{cb}=3.5)$

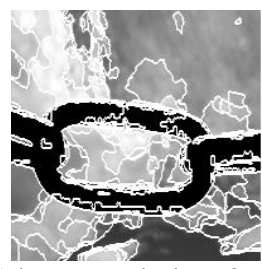

$(\mathrm{sb}=7$ and $\mathrm{cb}=3.5)$

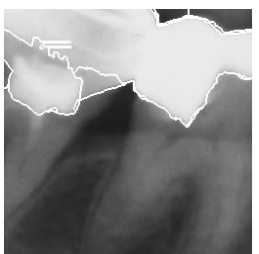

$(\mathrm{sb}=7$ and $\mathrm{cb}=6.5)$

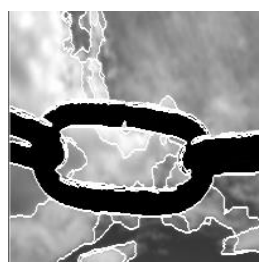

$(\mathrm{sb}=7$ and $\mathrm{cb}=6.5)$

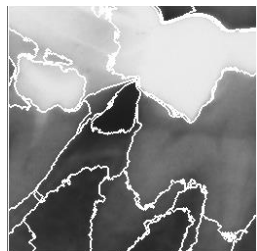

$(\mathrm{sb}=20$ and $\mathrm{cb}=4.5)$

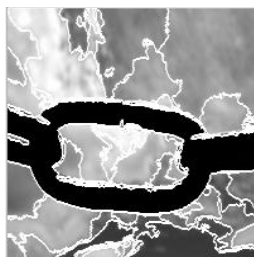

$(\mathrm{sb}=20$ and $\mathrm{cb}=4.5$

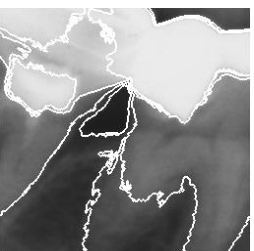

$(\mathrm{sb}=40$ and $\mathrm{cb}=6.5)$

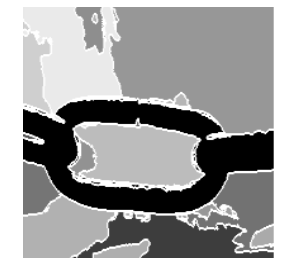

$(\mathrm{sb}=40$ and $\mathrm{cb}=6.5)$

Figure 11. Test initial segmentation with different parameter

\subsection{Experiment Analysis of Markers Process}

Interactive segmentation approach is very sensitive to the quality of marking and the number of user marker. This phenomenon has become a major concern in determining the number of given markers that will be used as a parameter [17]. Table 2 shows the number of markers based on the average value of ME on the natural and dental panoramic images. Number of marker 1, means that there is one marker for object and one marker for background show the smallest value of ME compared with two and three markers.

Table 2. Determination of Marker Number

\begin{tabular}{cccc}
\hline \multirow{2}{*}{ Images } & \multicolumn{4}{c}{ Number of Markers \& Misclassification Error (\%) } \\
& 1 & 2 & 3 \\
\hline Natural & 2.04 & 5.03 & 5.07 \\
Dental Panoramic & 5.47 & 8.82 & 10.31 \\
\hline
\end{tabular}

\subsection{Experiment Analysis of Fuzzy Region Merging}

Binary region merging (BRM) approach, as proposed [10] is not so effective for images that have a lot of ambiguous regions. The ambiguous region will lead to over-segmentation because there are some regions that have two values of information, both as background and object. To overcome this problem, this study propose fuzzy similarity measurements to find the greatest similarity value for the ambiguous region. Figure 12 illustrates the differences of segmentation result in the binary region merging (BRM) and the proposed fuzzy region merging (FRM). In Figure 12(b), we can see that each region has only a probability value of 0 and 1, in contrast to proposed method in Figure 12(c) where the value of each region ranged between 0 and 1.

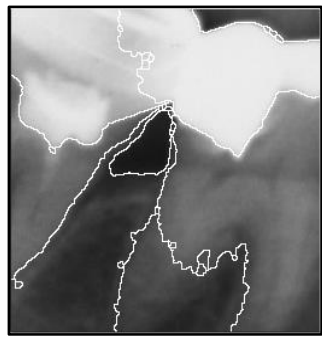

(a)

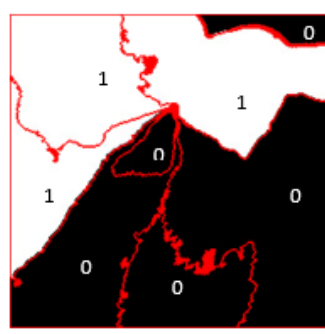

(b)

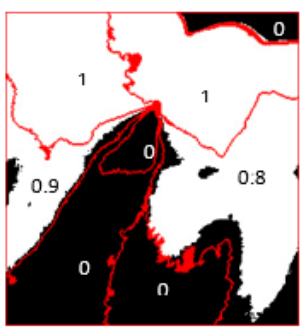

(c)

Figure 12. (a) Initial segmentation (b) Binary Region Merging, (c) Fuzzy Region Merging

\section{CONCLUSIONS}

Fuzzy Region Merging Using Fuzzy Similarity Measurement on Image Segmentation (Wawan Gunawan) 
In this study, we propose a new strategy for region merging process using fuzzy similarity measurement for image segmentation. Mean-shift algorithm was implemented to get initial segmentation. In the marking process, user give marker for the appropriate object and background region. Our contribution of this research is to separate ambiguous regions in the image using fuzzy similarity measurement. Based on the experimental results on the natural and dental panoramic images, the proposed method has successfully segmented the images with an average value of misclassification error (ME) $5.47 \%$ and $1.96 \%$, respectively. The proposed method only measures information from the gray level features and membership function. Therefore, combining information from other features such as spatial information, texture, and shape for region merging process can be developed further in order to obtain more accurate segmentation results.

\section{REFERENCES}

[1] D. A. Forsyth and J. Ponce, "Computer Vision: A Modern Approach,” Prentice Hall, 2002.

[2] T. Pavlidis, "Structural Pattern Recognition," Berlin Heidelberg: Springer-Verlag, 1977.

[3] A. Z. Arifin, et al., "Region Merging Strategy Using Statistical Analysis for Interactive Image Segmentation on Dental Panoramic Radiographs," International Review on Computers and Software, vol/issue: 12(1), pp. 63-74, 2017.

[4] N. Kamaruddin, et al., "Local region-based acm with fractional calculus for boundary segmentation in images with intensity inhomogeneity," Malaysian Journal of Computer Science, vol/issue: 29(2), pp. 124-144, 2016.

[5] C. Science and A. Pradesh, "Image Segmentation Based on Doubly Truncated Generalized Laplace Mixture Model and K Means Clustering," International Journal of Electrical and Computer Engineering, vol/issue: 6(5), pp. 2188-2196, 2016.

[6] K. Haris, et al., "Hybrid image segmentation using watersheds and fast region merging," IEEE Transactions on Image Processing, vol/issue: 7(12), pp. 1684-1699, 1998.

[7] H. Yao, et al., "An improved K-means clustering algorithm for fish image segmentation," Mathematical and Computer Modelling, vol/issue: 58(3-4), pp. 790-798, 2013.

[8] K. McGuinness and N. E. O'Connor, "A comparative evaluation of interactive segmentation algorithms," Pattern Recognition, vol/issue: 43(2), pp. 434-444, 2010.

[9] S. Hore, et al., "An Integrated Interactive Technique for Image Segmentation using Stack based Seeded Region Growing and Thresholding," International Journal of Electrical and Computer Engineering, vol/issue: 6(6), pp. $2773,2016$.

[10] J. Ning, et al., "Interactive image segmentation by maximal similarity based region merging," Pattern Recognition, vol/issue: 43(2), pp. 445-456, 2010.

[11] P. Salembier and L. Garrido, "Binary partition tree as an efficient representation for image processing, segmentation, and information retrieval," IEEE Transactions on Image Processing, vol/issue: 9(4), pp. 561-576, 2000.

[12] A. S. Sankoh, et al., "Extracted Pixels Similarity Features (EPSF) using Interactive Image Segmentation Techniques," International Journal of Computer Applications, vol. 136, pp. 1-8, 2016.

[13] A. Z. Arifin, et al., "Image thresholding using ultrafuzziness optimization based on type II fuzzy sets," IEEE International Conference on Instrumentation, Communications, Information Technology, and Biomedical Engineering (ICICI-BME), pp. 1-6, 2009.

[14] G. Q. O. Pratamasunu, et al., "Image thresholding based on index of fuzziness and fuzzy similarity measure," IEEE 8th International Workshop on Computational Intelligence and Applications (IWCIA), pp. 161-166, 2015.

[15] R. Dong, et al., "Interactive image segmentation with color and texture information by region merging," Chinese Control and Decision Conference (CCDC), vol/issue: 1(3), pp. 777-783, 2016.

[16] R. Indraswari, et al., "Teeth segmentation on dental panoramic radiographs using decimation-free directional filter bank thresholding and multistage adaptive thresholding," IEEE International Conference on Information \& Communication Technology and Systems (ICTS), pp. 49-54, 2015.

[17] M. Jian and C. Jung, "Interactive image segmentation using adaptive constraint propagation," IEEE Transactions on Image Processing, vol/issue: 25(3), pp. 1301-1311, 2016. 